\title{
Laminoplasty versus Laminectomy and Fusion for Multilevel Cervical Spondylotic Myelopathy
}

\author{
Barrett I. Woods MD, Justin Hohl MD, \\ Joon Lee MD, William Donaldson III MD, \\ James Kang MD
}

Published online: 19 November 2010

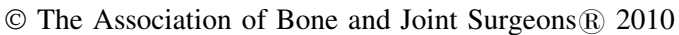

\begin{abstract}
Background Cervical spondylotic myelopathy is increasingly prevalent in the elderly and is the leading cause of spinal cord dysfunction in this population. Laminectomy with fusion and laminoplasty halt progression of myelopathy in these patients; however, both procedures have well-documented complications and associated morbidity and it is unclear which might be most advantageous. Questions/purposes We therefore compared the pain, function and alignment of patients who underwent laminectomy with fusion to those with laminoplasty for the treatment of multilevel cervical spondylotic myelopathy. Methods We performed a retrospective matched cohort analysis on all 121 patients from 2002 to 2007 who underwent laminectomy with fusion (82) or laminoplasty (39) for multilevel cervical spondylotic myelopathy. We determined change in preoperative and postoperative
\end{abstract}

Each author certifies that he or she has no commercial associations (eg, consultancies, stock ownership, equity interest, patent/licensing arrangements, etc) that might pose a conflict of interest in connection with the submitted article.

Each author certifies that his or her institution approved or waived approval for the human protocol for this investigation and that all investigations were conducted in conformity with ethical principles of research.

This work was performed at The Ferguson Lab for Orthopaedic and Spine Research, University of Pittsburgh Medical Center.

B. I. Woods, J. Hohl, J. Lee, W. Donaldson III, J. Kang

Department of Orthopaedic Surgery, University of Pittsburgh

Medical Center, Pittsburgh, PA, USA

J. Kang $(\square)$

The Ferguson Lab for Orthopaedic and Spine Research,

200 Lothrop Street, EBST 1641, Pittsburgh,

PA 15213, USA

e-mail: kangjd@upmc.edu sagittal alignment using Cobb measurement, development of junctional stenosis, and subjective improvements in pain and gait. Complications were recorded for both cohorts.

Results The majority of patients in both cohorts reported improvements in pain and gait postoperatively. There were seven complications in the laminectomy and fusion cohort (9\%) with two patients requiring formal revision surgery $(2 \%)$. There were five complications in the laminoplasty cohort (13\%) with two formal revision procedures (5\%).

Conclusions Patients in both the laminectomy with fusion and laminoplasty cohorts reported similar functional improvements after treatment for cervical spondylotic myelopathy. Prospective randomized control trials are needed to determine whether one procedure is truly superior.

Level of Evidence Level IV, therapeutic study. See Guidelines for Authors for a complete description of levels of evidence.

\section{Introduction}

Cervical spondylotic myelopathy (CSM) is the leading cause of spinal cord dysfunction in the elderly population [22]. Myelopathy results from cord compression from degenerative or congenital processes that usually lead to progressive and stepwise deterioration of neurologic function [5]. However, the early detection of this disorder can be challenging due to its insidious onset, initial subtle clinical findings, and presence of concomitant comorbidities in this elderly population. Due to the progressive neurologic deterioration associated with this disorder, a large percentage of these patients require operative intervention that decompresses the spinal cord to prevent progression and potentially reverse resultant myelopathy $[2,8,26]$. 
The surgical management of CSM has evolved considerably over the past 40 years; however, no surgical treatment is without associated morbidity. Laminectomy was initially regarded as the gold standard treatment of multilevel cervical myelopathy due to the extensive decompression this procedure afforded. However, welldocumented complications such as postlaminectomy kyphosis, segmental instability, and subsequent neurologic deterioration have led to the limited indication of laminectomy alone as a surgical option for this disease process $[3,6,11,20]$. Laminectomy was eventually augmented to include posterior fusion, which addressed the resultant instability incurred with removal of the posterior elements and has led to lower rates of kyphosis and segmental instability $[10,18,21]$. However, fusion of the cervical spine results in alteration of normal cervical biomechanics, as axial and rotational forces are no longer physiologically distributed to subjacent spinal structures. The alterations to force distribution following fusion procedures of the cervical spine have been associated with increased rates of adjacent segment degeneration $[4,16]$.

Laminoplasty was developed in Japan and allowed extensive cord decompression while preserving motion with less substantial alteration to the natural biomechanics of the cervical spine. Yet laminoplasty is contraindicated in patients with neutral or kyphotic alignment and has been associated with mechanical neck pain [1, 13, 23]. Despite major strides in the surgical management of multilevel CSM, our current options are not without morbidity.

In one previous retrospective study, laminectomy with fusion was associated with a higher complication rate and lower patient satisfaction than laminoplasty [12]. However, the small sample size did not provide power to draw definitive conclusions. While several studies demonstrate favorable neurologic recovery after both laminectomy with fusion $[9,14,15,20]$ and laminoplasty $[1,7,16,27]$ in patients with multilevel CSM, the superiority of one over the other is a subject of controversy in the literature [18, 24, 28].

We therefore compared (1) changes in preoperative sagittal alignment of the cervical spine and junctional segments in patients who received laminectomy with fusion to those with laminoplasty for the treatment of multilevel CSM; (2) subjective functional improvement (pain and gait) after surgery in these two cohorts; and (3) rate of major complications following surgery in these two cohorts.

\section{Patients and Methods}

We retrospectively reviewed all 255 patients who underwent laminectomy with fusion (185) or laminoplasty (70) for the presence of multilevel CSM or myeloradiculopathy in the practice of senior author (JDK) over a 5 year period (2002-2007). We included patients with (1) clearly documented physical examination findings consistent with progressive myelopathy or myeloradiculopathy who failed nonoperative measures; and (2) radiographic confirmation of cord compression at three or more cervical levels. We excluded 112 patients whose presenting complaint was axial neck pain alone, those with fractures, segmental cervical instability, tumor, metabolic disorders, or previous history of cervical spine surgery, patients who had concurrent anterior cervical spine procedures or those with combined laminoplasty and fusion. This left 143 patients; 22 were subsequently excluded due to an incomplete medical record or inappropriate radiographic follow up. Thus, 121 patients were included in this retrospective matched cohort analysis, 82 with laminectomy and fusion and 39 with laminoplasty. No patients were recalled specifically for this study; all data were obtained from medical records or radiographs. Information collected included patient age at time of surgery, gender, preoperative ambulatory status, physical examination findings, comorbidities, length of radiographic followup, postoperative complications, and ambulatory status. All major complications following surgery were recorded in each cohort. The matched cohort data illustrates age, gender, symptoms, duration, and average radiographic followup. All patients included in the study had myelopathy; however 35 and $33 \%$ respectively also had evidence of radicular symptoms on physical exam (Table 1). Minor complications were considered any deviation from normal postoperative course which may or may not require pharmacological intervention (persistent nausea, dehydration, electrolyte imbalance, etc.) and were not recorded. Minimum follow up was 6.7 months, with an average of 24 months (6.7 to 31.6 months) for the laminectomy/fusion group and 24 months (7.3 to 29.8 months) for the laminoplasty group.

Indications and contraindications for each procedure were determined by the senior author and surgeon (JDK). Relative indications for laminoplasty were the presence of multilevel disease, lordotic sagittal alignment in the

Table 1. Matched cohort data

\begin{tabular}{llllll}
\hline Procedure & $\begin{array}{l}\text { Age } \\
\text { (years SD) }\end{array}$ & Gender & $\begin{array}{l}\text { Myelopathic/myeloradicular } \\
\text { symptoms }\end{array}$ & $\begin{array}{l}\text { Duration of symptoms } \\
\text { (months) }\end{array}$ & $\begin{array}{l}\text { Average radiographic } \\
\text { follow up (months) }\end{array}$ \\
\hline Laminectomy with fusion & $64(10.7)$ & $49 \mathrm{M} / 32 \mathrm{~F}$ & $64 \% / 35 \%$ & $16(11.2)$ & $23.99(9.91)$ \\
Laminoplasty & $60(12.5)$ & $25 \mathrm{M} / 14 \mathrm{~F}$ & $67 \% / 33 \%$ & $14(8.3)$ & $23.81(5.98)$ \\
\hline
\end{tabular}


absence of large anterior osteophytes, or substantial axial neck pain. Relative indications for laminectomy and fusion included patients with multilevel disease and lordotic or neutral sagittal alignment in the absence of large anterior osteophytes. The only absolute contraindication for laminoplasty was a kyphotic cervical spine sagittal alignment or severe mechanical neck pain. Patients with substantial myeloradicular symptoms underwent laminoplasty with the opening wedge correlating to the symptomatic side, or in patients with laminectomy and fusion, a corresponding foraminotomy was performed.

All surgery was performed by one surgeon (JDK). Each procedure was tailored to the patient's individual pathology and the decompressed segments correlated with radiographic findings. The standard posterior approach was used for both procedures. Fifty-three patients received a C3-C7 laminectomy and fusion. Seven patients received a $\mathrm{C} 4-\mathrm{C} 7$ laminectomy with $\mathrm{C} 3-\mathrm{C} 7$ fusion. Five patients received C3-C7 laminectomy with $\mathrm{C} 3-\mathrm{T} 1$ fusion. The remaining 17 patients received a variation of different cervical segment decompression and fusion (eg, C3-C5 laminectomy with C2-C6 fusion). The decision to fuse segments not decompressed was made intraoperatively based on bone quality and stability of the construct. Bicortical screw placement was used in patients in which unicortical fixation was inadequate. All patients received iliac crest bone graft to facilitate bone healing. In laminectomy and fusion constructs that extended to $\mathrm{C} 7$, there was an effort not to take all of the lamina, removing only the cranial half, thus keeping the interspinous and supraspinous ligaments attached to the C7 spinous process. Salvaging the C7 ligaments kept the $\mathrm{C} 7-\mathrm{T} 1$ junction more stable, thus hopefully decreasing the incidence of junctional degeneration and axial neck pain. Additionally, pedicle screws were placed in $\mathrm{C} 7$ vertebrae in which the obliquity of the lateral masses was deemed too substantial for the stable screw placement; all other levels received lateral mass fixation.

Patients in the laminoplasty group all received C3-C7 laminoplasty using Mitek (Norwood, MA) suture anchor fixation [19]. The direction of the laminoplasty was based on the patient's symptoms and physical examination preoperatively. Lateral decompression was performed carefully, stopping just medial to the zygopophyseal joint to preserve the capsule.

Postoperative protocols were standard for both surgical groups and included placement of semirigid or rigid cervical spine collars 6 to 12 weeks postoperatively for the laminectomy/fusion and laminoplasty groups, respectively.

All patients were scheduled for followup in clinic 1, 3, 6,12 , and 24 months after surgery. We obtained MRI scans postoperatively when clinically indicated as determined by the senior surgeon to access patency of decompression, and all patients received standard flexion extension, neutral lateral, and AP cervical spine films at 1 and 6 months' followup. Additionally, the senior surgeon made a subjective assessment gait. Patient function was assessed at postoperative visits through detailed physical examinations, gait analysis, and patient interaction with senior author and surgeon. Overall pain and gait improvement postoperatively were determined by patients' responses on a 5-point scale at least 6 months after surgery. This scale included the following response to improvement with the procedure as very poor, poor, fair, good, or very good on an ascending scale from 1 to 5 . A positive response to surgery for each criteria pain and gait was recorded in patients who stated improvement in that area was good (4) or very good (5).

One of us (BIW) reviewed all radiographs. Preoperative cervical spine films were measured for segmental listhesis and cervical lordosis from $\mathrm{C} 2-\mathrm{C} 7$ using the Cobb method and previously described Ishihara index [18]. Postoperative films were analyzed for hardware failure (broken or retracting screws), lucent areas around the construct, segmental listhesis, and cervical lordosis using the Cobb method of C2-C7. Cobb angles were also obtained on the supra- or subjacent level to be decompressed and compared with postoperative films.

First, for the statistical analysis of the radiographic measurements (Cobb and junctional Cobb angles), a twoway, mixed-measures ANOVA was performed. The degree of Cobb angle correction obtained postoperatively for the laminectomy with fusion and laminoplasty cohorts was an independent variable of analysis. Student's $t$ tests were performed post hoc with Bonferroni correction $(\mathrm{p}<$ 0.0125). We used a Student's t test to determine the difference between Ishihara scores in the two groups and a Pearson's chi square test to determine differences in subjective clinical improvement between cohorts. A Student's $t$ test was performed to determine whether the rates of complications present in the two groups were different. We used SPSS ${ }^{\circledR}$ (Version 16.0; SPSS Inc, Chicago, IL) for all analyses.

\section{Results}

The laminectomy with fusion and laminoplasty cohorts had similar $(\mathrm{p}=0.72)$ preoperative lordotic alignment with Ishihara indices of 0.13 and 0.17 , respectively. Postoperatively, the mean cervical sagittal alignment remained lordotic for both cohorts, although the laminectomy with fusion group had a larger alteration from preoperative sagittal alignment, losing on average $2.57^{\circ}$ of lordosis, while the laminoplasty group actually gained $0.57^{\circ}$ of lordosis. We found no differences in preoperative alignment (Ishihara, Cobb) between the laminectomy fusion and 
Table 2. Pre- and postoperative radiographic analysis

\begin{tabular}{|c|c|c|c|c|c|}
\hline \multirow[t]{2}{*}{ Procedure } & \multirow{2}{*}{$\begin{array}{l}\text { Preoperative sagittal } \\
\text { alignment (Ishihara) }\end{array}$} & \multicolumn{2}{|c|}{ Decompressed segments (Cobb) } & \multicolumn{2}{|c|}{ Junctional segments (Cobb) } \\
\hline & & Preoperative & Most recent & Preoperative & Most recent \\
\hline Laminectomy Fusion & 0.13 & $10.16 \mathrm{~L}$ & $7.59 \mathrm{~L}^{*}$ & $3.98 \mathrm{~L}$ & $5.08 \mathrm{~K}$ \\
\hline Laminoplasty & 0.17 & $12.05 \mathrm{~L}$ & $12.62 \mathrm{~L}$ & $2.92 \mathrm{~L}$ & $3.79 \mathrm{~K}$ \\
\hline
\end{tabular}

* Denotes statistical significance.

laminoplasty cohorts. There was a significant change $(\mathrm{p}<0.0125)$ in sagittal alignment postoperatively in the laminectomy and fusion group (Table 2).

The junctional segment for both cohorts became more kyphotic after decompression. In all but four patients the junctional segment represented the corresponding subadjacent level. There was no difference $(\mathrm{F}=3.809$, $\mathrm{p}=0.053)$ in junctional kyphosis between the laminectomy with fusion or laminoplasty cohort.

Both cohorts showed improvement in gait and pain after surgery. The patients who underwent laminectomy with fusion tended to have better overall functional results with regard to pain and gait than the laminoplasty cohort. A total of 58 patients in the laminectomy fusion group and 26 patients in the laminoplasty group reported good or very good response to improvement in gait. A total of 60 patients in the laminectomy with fusion group and 23 in the laminoplasty group reported good or very good improvement in pain. We found no differences between the two groups for gait $(\mathrm{p}=0.674)$ or pain $(\mathrm{p}=0.141)$ postoperatively following either procedure. Patients classified as improved recorded a 4 (good) or 5 (very good) response on a subjective questionnaire for gait or pain at least 6 months postoperatively (Table 3).

All complications recorded in this study were considered major (associated with increased morbidity and of clinical relevance). Overall, complication rates were not different $(\mathrm{p}=0.087)$ between cohorts. Seven complications $(9 \%)$ were recorded in the laminectomy with fusion group and included chronic pain, dysphagia, pseudarthrosis, infection, junctional stenosis, and progressive kyphosis after hardware failure. Of the patients who developed chronic pain postoperatively, both reported substantial iliac crest bone graft donor site morbidity. Three patients in the laminectomy fusion cohort had reoperation, two of whom underwent formal revision surgery (Fig. 1). The one patient who had reoperation but not revision had persistent posterior wound drainage and subsequent dehiscence. He was taken back to the operating room for irrigation and débridement with placement of a wound vacuum. He eventually required a trapezius flap for soft tissue coverage.

Of the other two patients in the laminectomy fusion group who had formal revision, one had progressive kyphotic deformity after hardware failure and the other
Table 3. Subjective clinical improvement postoperatively

\begin{tabular}{lll}
\hline Procedure & Gait & Pain \\
\hline Laminectomy Fusion & $71 \%$ & $74 \%$ \\
Laminoplasty & $68 \%$ & $60 \%$ \\
\hline
\end{tabular}

developed symptomatic junctional stenosis. In the laminoplasty cohort, there were five complications (13\%), three of which related to chronic mechanical neck pain. Two patients who had revision surgery: one who developed recurrent stenosis due to large anterior osteophytes and one who had persistent and progressive radiculopathy postoperatively (Fig. 2).

The rate of revision surgery was similar $(\mathrm{p}=0.074)$ between the two groups. Additionally, there were no infections in the laminoplasty cohort. The most common complaint postoperatively for the laminoplasty group was neck pain $(32 \%)$; however, only $8 \%$ of these patients had substantial debilitating neck pain at final followup, as compared to $2 \%$ of the laminectomy fusion group $(\mathrm{p}=0.066)$. The most common complaint for the laminectomy with fusion group was neck stiffness (12\%) (Table 4; Fig. 3).

\section{Discussion}

The cervical spine can be adequately decompressed using anterior or posterior procedures. The decision as to which way to address the resultant CSM is based upon several factors, including patient health, previous spinal surgery, sagittal alignment, location of pathology, presence of multilevel disease, preoperative function, and pain. Anterior procedures (anterior cervical discectomy and fusion, corpectomy) have several advantages, including a musclesparing approach, low wound complication rates, and ability to directly address kyphotic deformity, whereas posterior procedures may be better suited to address multilevel cervical disorders [17, 25, 29].

There are a number of limitations to this retrospective review. First, our radiographic analysis is relatively short (mean, 24 months for both groups) and longer followup will be required to determine the durability of our findings. Second, we had no validated outcome measures and rather 
Fig. 1A-C Images illustrate the case of a patient who initially underwent $\mathrm{C} 3-\mathrm{C} 7$ laminectomy and fusion due to progressive myeloradiculopathy. (A) His course was complicated by junctional stenosis, progressive cervicothoracic kyphosis, and recurrent neurologic symptoms. (B) Lateral radiographs before and $(\mathbf{C})$ after show revision junctional laminectomies with $\mathrm{C} 2-\mathrm{T} 2$ posterior cervical fusion.
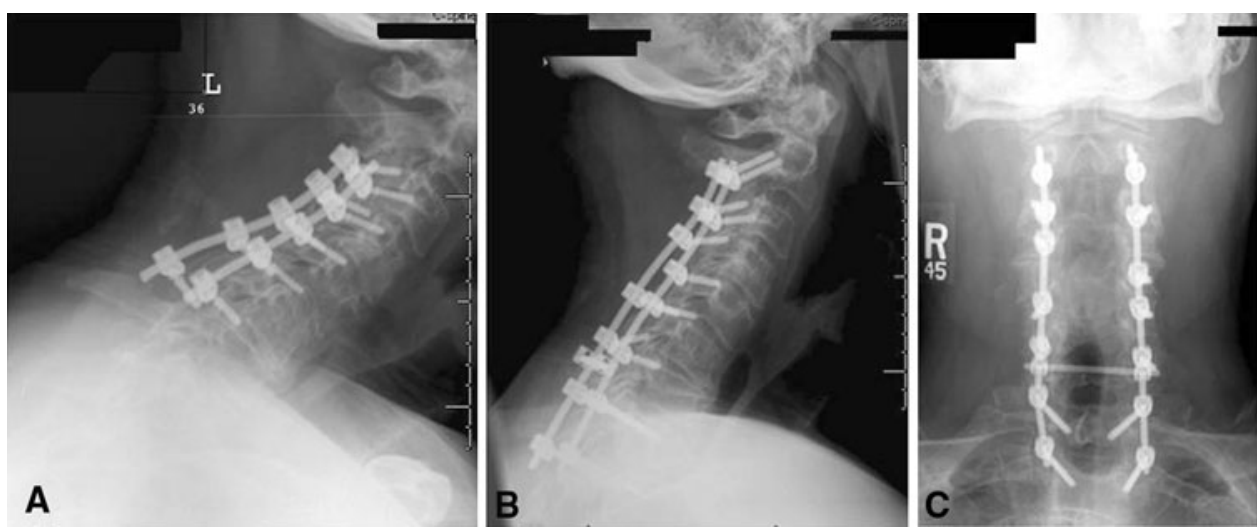

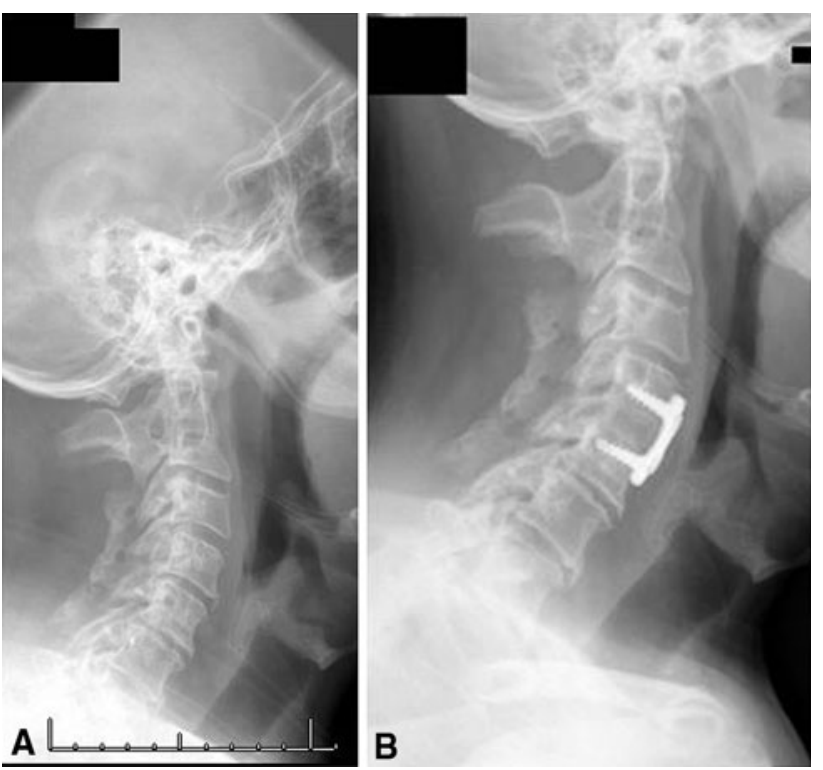

Fig. 2A-B Images illustrate the case of a 51-year-old man who had C3-C7 laminoplasty for multilevel cervical stenosis. (A) Two years postoperatively, he developed uncovertebral spurs and recurrent radiculopathy. (B) He underwent C4-C5 anterior cervical discectomy and fusion with eventual relief of radicular symptoms.

relied on subjective analysis. Considering the subjectivity of pain and gait validated measures would more accurately represent these clinical parameters. Third, the senior author who performed the surgery also made all postoperative assessments and was not blinded. Additionally it is noted that physical exam was used to delineate myeloradiculopathy from axial neck pain, however no standard protocol for that assessment was found in the medical record. Diagnosis and treatment decisions were made solely by the senior author. Fourth, although no patients were lost to followup, 22 patients or $15 \%$ of patients who met inclusion criteria were eliminated due to incomplete medical records as most lacked the subjective data needed to determine their outcome. The inclusion of these data if available may have altered the trends observed or resulted in notable
Table 4. Complications following surgery

\begin{tabular}{|c|c|c|c|}
\hline $\begin{array}{l}\text { Laminectomy } \\
\text { fusion }\end{array}$ & $\begin{array}{l}\text { \# of } \\
\text { patients }\end{array}$ & Laminoplasty & $\begin{array}{l}\# \text { of } \\
\text { patients }\end{array}$ \\
\hline Chronic pain & $2(2 \%)$ & Chronic pain & $3(8 \%)$ \\
\hline Dysphagia & $1(1 \%)$ & Recurrent stenosis & $1 *(3 \%)$ \\
\hline Pseudoarthrosis & $1(1 \%)$ & $\begin{array}{l}\text { Persistent } \\
\quad \text { radiculopathy }\end{array}$ & $1 *(3 \%$ \\
\hline Infection & $1(1 \%)$ & & \\
\hline Junctional stenosis & $1 *(1 \%)$ & & \\
\hline Progressive kyphosis & $1 *(1 \%)$ & & \\
\hline Revision surgery & $2(2 \%)$ & Revision surgery & $2(5 \%)$ \\
\hline
\end{tabular}

* Denotes revision required.

findings. Fifth, statistical significance was not achieved on many parameters likely due to the study being underpowered. Thus, it is difficult to draw definitive conclusions from this data set of 121 patients, although compared to a previously published report [12], it is relatively large. Finally although immediate post operative protocols were standardized there was no record of the physiotherapy these patients received postoperatively which could have positively or negatively influenced their outcome and subject assessment of their symptoms.

Laminectomy with fusion and laminoplasty have emerged as the two most frequently used procedures for addressing multilevel CSM, as both can halt myelopathic progression and improving patients symptoms [7, 9, 12, 24, 27]. However, each is associated with specific morbidity and controversy exists as to which surgical intervention should be the standard of care in patients with multilevel CSM and maintained physiologic sagittal alignment (cervical lordosis). There have been few retrospective cohort studies comparing outcomes in patients who have multilevel CSM and received laminectomy with fusion or laminoplasty and no prospective randomized control trials to the authors' knowledge. Heller et al. [12] performed a retrospective matched cohort analysis of patients with multilevel cervical myelopathy who had 

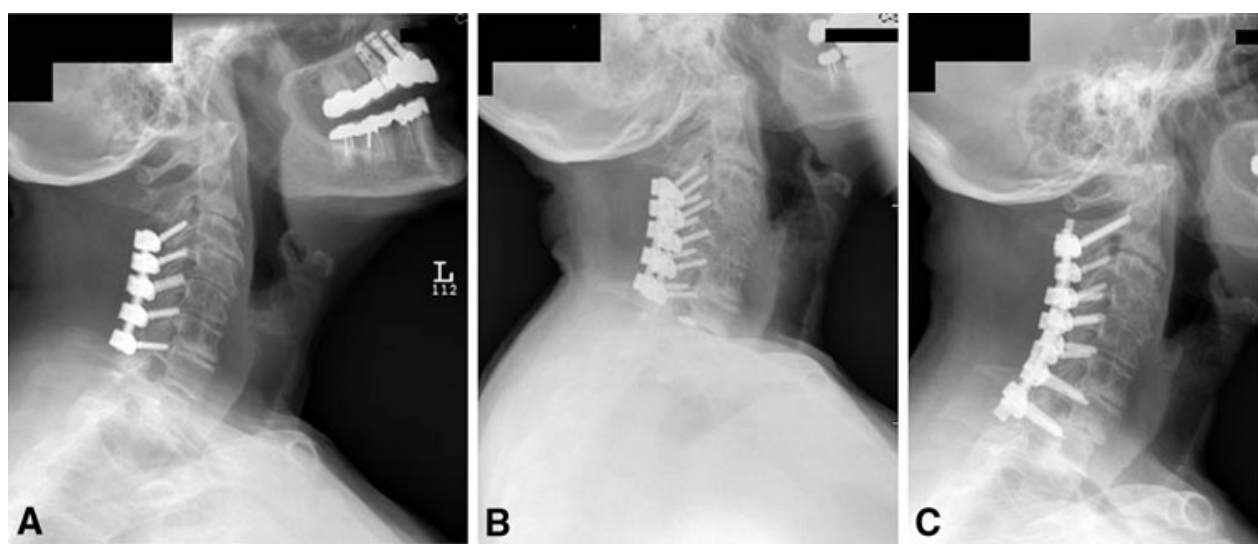

Fig. 3A-C Images illustrate the case of a 78-year-old woman who underwent $\mathrm{C} 3-\mathrm{C} 7$ laminectomy and fusion for multilevel cervical stenosis due to degenerative disc disease. (A) A postoperative radiograph shows appropriately placed hardware and lordotic sagittal alignment. (B) Two years later, the patient presented with recurrent

either laminectomy with fusion or laminoplasty and found both procedures provided functional improvement; however, only laminoplasty consistently halted myelopathic progression, had higher patient satisfaction, and had a substantially lower complication rate. Other studies have not consistently shown laminoplasty to be superior to laminectomy and fusion $[9,10,14,17,18,22,23,30]$. In this retrospective matched cohort analysis, we compared (1) changes in preoperative sagittal alignment of the cervical spine and junctional segments in patients who received laminectomy with fusion to those with laminoplasty for the treatment of multilevel CSM; (2) subjective functional improvement (pain and gait); and (3) rate of major complications after surgery in these two cohorts.

Preoperative sagittal alignment of the spine has prognostic implications when contemplating surgical decompression for myelopathic patients. With normal physiologic (lordotic) alignment, after decompression, the spine cord can drift posteriorly away from compressive pathology. Loss of physiologic alignment impairs the dorsal migration of the cord and has resulted in inferior neurologic recovery after surgery [17]. Thus, patient selection preoperatively and maintenance of physiologic alignment postoperatively may have important implications on clinical outcomes. The results from our radiographic analysis (Table 2) illustrate preoperatively both groups had physiologic sagittal alignments, and thus patients were appropriately matched. The only radiographic finding that was different postoperatively was some loss of cervical lordosis after laminectomy with fusion. Additionally, we observed a trend for change in junctional kyphosis in the laminectomy fusion group from lordosis to kyphosis. From this data, it can be concludedd laminectomy with fusion may have a more profound effect on the normal biomechanics of the spine and the loss of symptoms and had substantially progressed multilevel disc degeneration. (C) The patient underwent revision junctional laminectomies with $\mathrm{C} 2-\mathrm{T} 1$ posterior fusion and had functional improvement postoperatively.

lordosis seen postoperatively may be due to the development of more pronounced junctional kyphosis. This finding did not correlate with clinical or subjective improvement in this data set. Due to the relatively short followup for both surgical groups, recurrent symptoms for progressive junctional change or hardware failure in either group may not present over this time course.

Subjective clinical improvement occurred in the majority of both cohorts with improvement in gait and pain for the laminectomy with fusion cohort slightly exceeding that of the laminoplasty cohort $(71 \%, 74 \%$, versus $68 \%$, $60 \%$ ). There was no difference in regard to improvement in gait or pain with the procedure; however, the laminectomy/ fusion cohort illustrated a trend toward slightly better functional results. This differs from the data set of Heller et al. [12], which showed higher patient satisfaction and subjective clinical improvement (strength, dexterity, numbness, pain, gait) when compared to laminectomy with fusion. From a summation of the current literature, it is clear there is no consensus as to the superiority of one procedure over the other in regard to the subjective clinical improvement in gait, pain, or patient satisfaction $[10,13$, 23]. However, in our study and in other published reports, laminoplasty was associated with less improvement in neck pain symptoms and more axial neck pain postoperatively than laminectomy and fusion $[9,22,28,29]$.

Historically, infection and soft tissue complications have been higher in the laminectomy with fusion group and this is attributed to longer intraoperative times, largevolume blood loss, and more aggressive soft tissue dissection [4]. The rate of infection of our laminectomy with fusion series was $1.1 \%$, which is lower than other published reports [15]. There were no infections in the laminoplasty cohort. The most important postoperative complication 
experienced by patients in the laminoplasty group was persistent chronic pain. The laminectomy with fusion and laminoplasty cohorts both had a large number of patients reporting mechanical neck pain 3 months after surgery, $24 \%$ and $32 \%$, respectively. However, only a small percentage of those patients reported persistent and debilitating pain at most recent examination $(2 \%$ of the laminectomy with fusion patients and $8 \%$ of the laminoplasty patients). Previous studies have documented persistent neck pain in up $25 \%$ of laminoplasty patients [13]. From this data set, the rate of major complications was $8 \%$ for the laminectomy and fusion cohort and $13 \%$ for the laminoplasty. Additionally, there was no statistical difference in revision rates between laminectomy/fusion $(2 \%)$ and laminoplasty (5\%). It should be noted neck stiffness was not included as a complication after laminectomy/fusion despite being a complaint in 10 patients (12\%). The rationale behind this is that stiffness postoperatively is expected because, with fusion for multilevel CSM, construct stability and absence of motion of the involved segments may improve the chance of vascular and neurologic recovery. Stiffness does increase the morbidity of this procedure despite not being considered a complication, especially in the younger patients, as it can affect activities of daily living such as driving a vehicle.

Both laminectomy with fusion and laminoplasty decompress the cervical spine and provide clinical improvement to patients with CSM. There was no difference in functional outcomes between these groups, although laminectomy with fusion had a trend toward improved functional outcomes. The laminectomy cohort showed trends toward lower complication, chronic pain, and revision rates than the laminoplasty cohort. Laminectomy with fusion and laminoplasty have both illustrated efficacy in halting the myelopathic progression of patients with multilevel CSM, but prospective randomized control trials are needed to determine if one procedure is superior to the other. Additionally, a prospective design could address the weaknesses of this study. Current treatment algorithms must take into account surgeon experience with each procedure, global cervical sagittal alignment, symptoms, and location of pathology when choosing between these two procedures.

Acknowledgments We thank Cara Davies and Robert Hartman for their vital contributions with data collection and statistical analysis, which aided in the completion of this manuscript.

\section{References}

1. Baba H, Uchida K, Maezawa Y, Furusawa N, Azuchi M, Imura S. Lordotic alignment and posterior migration of the spinal cord following en bloc open-door laminoplasty for cervical myelopathy: a magnetic resonance imaging study. J Neurol. 1996;243:626-632.

2. Bucciero A, Vizioli L, Carangelo B, Tedeschi G. MR signal enhancement in cervical spondylotic myelopathy: correlation with surgical results in 35 cases. J Neurosurg Sci. 1993;37: 217-222.

3. Butler JC, Whitecloud TS. Postlaminectomy kyphosis: causes and surgical management. Orthop Clin North Am. 1992;23: 505-511.

4. Cherubino P, Benazzo F, Borromeo U, Perle S. Degenerative arthritis of the adjacent spinal joints following anterior cervical spinal fusion: clinicoradiologic and statistical correlations. Ital J Orthop Traumatol. 1990;16:533-543.

5. Clarke E, Robinson PK. Cervical myelopathy: a complication of cervical spondylosis. Brain. 1956;79:483-510.

6. Dai L, Ni B, Yuan W, Jia L. Radiculopathy after laminectomy for cervical compression myelopathy. J Bone Joint Surg Br.1998;80: 846-849.

7. Edwards CC, Heller JG, Silcox DH. T-Saw laminoplasty for the management of cervical spondylotic myelopathy: clinical and radiographic outcome. Spine (Phila Pa 1976). 2000;25:17881794.

8. Fujiwara K, Yonenobu K, Ebara S, Yamashita K, Ono K. The prognosis of surgery for cervical compression myelopathy: an analysis of the factors involved. J Bone Joint Surg Br. 1989;71: 393-398.

9. Gok B, McLoughlin GS, Sciubba DM, McGirt MJ, Chaichana KL, Wolinsky JP, Bydon A, Gokaslan ZL, Witham TF. Surgical management of cervical spondylotic myelopathy with laminectomy and instrumented fusion. Neurol Res. 2009;31:1097-1101.

10. Gonzalez-Feria L, Peraita-Peraita P. Cervical spondylotic myelopathy: a cooperative study. Clin Neurol Neurosurg. 1975;78: 19-33.

11. Guigui P, Benoist M, Deburge A. Spinal deformity and instability after multilevel cervical laminectomy for spondylotic myelopathy. Spine(Phila Pa 1976) 1998;23:440-447.

12. Heller JG, Edwards CC, Murakami H, Rodts GE. Laminoplasty versus laminectomy and fusion for multilevel cervical myelopathy: an independent matched cohort analysis. Spine (Phila Pa 1976). 2001;26:1330-1336.

13. Hosono N, Yonenobu K, Ono K. Neck and shoulder pain after laminoplasty: a noticeable complication. Spine (Phila Pa 1976). 1996;21:1969-1973.

14. Houten JK, Cooper PR. Laminectomy and posterior cervical plating for multilevel cervical spondylotic myelopathy and ossification of the posterior longitudinal ligament: effects on cervical alignment, spinal cord compression, and neurological outcome. Neurosurgery. 2003;52:1081-1087; discussion 10871088.

15. Huang RC, Girardi FP, Poynton AR, Cammisa FP Jr. Treatment of multilevel cervical spondylotic myeloradiculopathy with posterior decompression and fusion with lateral mass plate fixation and local bone graft. J Spinal Disord Tech. 2003;16: $123-129$

16. Kato Y, Iwasaki M, Fuji T, Yonenobu K, Ochi T. Long-term follow-up results of laminectomy for cervical myelopathy caused by ossification of the posterior longitudinal ligament. J Neurosurg. 1998;89:217-223.

17. Kawakami M, Tamaki T, Iwasaki H, Yoshida M, Ando M, Yamada H. A comparative study of surgical approaches for cervical compressive myelopathy. Clin Orthop Relat Res. 2000:381;129-136.

18. Kumar VG, Rea GL, Mervis LJ, McGregor JM. Cervical spondylotic myelopathy: functional and radiographic long-term outcome after laminectomy and posterior fusion. Neurosurgery. 1999;44:771-777; discussion 777-778. 
19. Lee JY, Hanks SE, Oxner W, Tannoury C, Donaldson WF, Kang JD. Use of small suture anchors in cervical laminoplasty to maintain canal expansion: a technical note. J Spinal Disord Tech. 2007;20:33-35.

20. Lonstein JE. Post-laminectomy kyphosis. Clin Orthop Relat Res. 1977;128:93-100.

21. Maurer PK, Ellenbogen RG, Ecklund J, Simonds GR, Van Dam B, Ondra SL. Cervical spondylotic myelopathy: treatment with posterior decompression and Luque rectangle bone fusion. Neurosurgery. 1991;28:680-683; discussion 683-684.

22. Rao RD, Gourab K, David KS. Operative treatment of cervical spondylotic myelopathy. J Bone Joint Surg Am. 2006;88:16191640.

23. Ratliff JK, Cooper PR. Cervical laminoplasty: a critical review. J Neurosurg 2003;98:230-238.

24. Satomi K, Nishu Y, Kohno T, Hirabavashi K. Long-term followup studies of open-door expansive laminoplasty for cervical stenotic myelopathy. Spine (Phila Pa 1976). 1994;19:507-510.

25. Sodeyama T, Goto S, Mochizuki M, Takahashi J, Moriya H. Effect of decompression enlargement laminoplasty for posterior shifting of the spinal cord. Spine (Phila Pa 1976). 1999;24: 1527-1531; discussion 1531-1532.

26. Suri A, Chabbra RP, Mehta VS, Gaikwad S, Pandey RM. Effect of intramedullary signal changes on the surgical outcome of patients with cervical spondylotic myelopathy. Spine J. 2003; 3:33-45.

27. Tomita K, Kawahara N, Toribatake Y, Heller JG. Expansive midline T-saw laminoplasty (modified spinous process-splitting) for the management of cervical myelopathy. Spine (Phila Pa 1976). 1998;23:32-37.

28. Wang MY, Shah S, Green BA. Clinical outcomes following cervical laminoplasty for 204 patients with cervical spondylotic myelopathy. Surg Neurol. 2004;62:487-492; discussion 492-493.

29. Yonenobu K, Fuji T, Ono K, Okada K, Yamamoto T, Harada N. Choice of surgical treatment for multisegmental cervical spondylotic myelopathy. Spine (Phila Pa 1976). 1985;10:710-716.

30. Yonenobu K, Hosono N, Iwasaki M, Asano M, Ono K. Laminoplasty versus subtotal corpectomy: a comparative study of results in multisegmental cervical spondylotic myelopathy. Spine (Phila Pa 1976). 1992;17:1281-1284. 